\title{
PRISMS: An Integrated, Open-Source Framework for Accelerating Predictive Structural Materials Science
}

\author{
L.K. AAGESEN, ${ }^{1,2}$ J.F. ADAMS, ${ }^{1,3}$ J.E. ALLISON, ${ }^{1,13}$ W.B. ANDREWS, ${ }^{1}$ \\ V. ARAULLO-PETERS, ${ }^{1,4}$ T. BERMAN ${ }^{1}$ Z. CHEN ${ }^{5}$ S. DALY ${ }^{5}$ \\ S. DAS, ${ }^{6}$ S. DEWITT, ${ }^{1}$ S. GANESAN,${ }^{3,7}$ K. GARIKIPATI,${ }^{6}$ V. GAVINI, ${ }^{1,6}$ \\ A. GITHENS, ${ }^{1}$ M. HEDSTROM ${ }^{8}$ Z. HUANG,${ }^{1}$ H.V. JAGADISH, ${ }^{9}$ \\ J.W. JONES, ${ }^{1}$ J. LUCE,${ }^{1}$ E.A. MARQUIS, ${ }^{1}$ A. MISRA, ${ }^{1}$ D. MONTIEL ${ }^{1}$ \\ P. MOTAMARRI, ${ }^{6}$ A.D. MURPHY, ${ }^{1}$ A.R. NATARAJAN,${ }^{10}$ S. PANWAR, ${ }^{7}$ \\ B. PUCHALA ${ }^{1}$ L. QI ${ }^{1}$ S. RUDRARAJU,${ }^{6,11} \mathrm{~K}$. SAGIYAMA,${ }^{6}$ \\ E.L.S. SOLOMON,${ }^{1,12}$ V. SUNDARARAGHAVAN,${ }^{7}$ G. TARCEA, ${ }^{9}$ \\ G.H. TEICHERT, ${ }^{6}$ J.C. THOMAS, ${ }^{10}$ K. THORNTON, ${ }^{1}$ A. VAN DER VEN, ${ }^{10}$ \\ Z. WANG ${ }^{6}{ }^{\text {T. WEYMOUTH }}{ }^{1}{ }^{1}$ and C. YANG $^{1}$
}

1.-Materials Science and Engineering, University of Michigan, Ann Arbor, MI 48109, USA. 2.-Present Address: Idaho National Laboratory, Idaho Falls, ID 83415, USA. 3.-Present Address: Intel Corp, Hillsboro, OR 97124, USA. 4.-Present Address: Queen Mary University of London, London E1 4NS, UK. 5.-Mechanical Engineering, University of California Santa Barbara, Santa Barbara, CA 93106, USA. 6.-Mechanical Engineering, University of Michigan, Ann Arbor, MI 48105, USA. 7.-Aerospace Engineering, University of Michigan, Ann Arbor, MI 48109, USA. 8.-School of Information, University of Michigan, Ann Arbor, MI 48109, USA. 9.-Electrical Engineering and Computer Science, University of Michigan, Ann Arbor, MI 48109, USA. 10.-Materials Department, University of California Santa Barbara, Santa Barbara, CA 93106, USA. 11.- Present Address: Mechanical Engineering, University of Wisconsin, Madison, WI 53706, USA. 12.-Present Address: Engineering Systems Inc, Ann Arbor, MI 48108, USA. 13.—e-mail: johnea@umich.edu

The Center for Predictive Integrated Structural Materials Science (PRISMS Center) is creating a unique framework for accelerated predictive materials science and rapid insertion of the latest scientific knowledge into next-generation ICME tools. There are three key elements of this framework. The first is a suite of high-performance, open-source integrated multi-scale computational tools for predicting microstructural evolution and mechanical behavior of structural metals. Specific modules include statistical mechanics, phase field, crystal plasticity simulation and real-space DFT codes. The second is the Materials Commons, a collaboration platform and information repository for the materials community. The third element of the PRISMS framework is a set of integrated scientific "Use Cases" in which these computational methods are linked with experiments to demonstrate the ability for improving our predictive understanding of magnesium alloys, in particular, the influence of microstructure on monotonic and cyclic mechanical behavior. This paper reviews progress toward these goals and future plans.

\section{INTRODUCTION}

The materials field has benefited significantly from major advances in mechanistic understanding enabled by the development and application of computational methods. However, these advances have not translated into a general reduction in the time required to develop new materials. Multi-user, professional level software such as VASP and opensource codes such as Quantum ESPRESSO ${ }^{1}$ have been developed in Europe where long-term funding for professional level software development has been a priority. In contrast, the materials science software developed at U.S. universities has largely been designed for use by individual research groups and close collaborators. This fragmentation and the 
lack of a framework for building integrated platforms, software and services have limited the evolution and wide availability of advanced predictive approaches. While the long-range goal of making the materials field more computationally oriented and holistic has been well articulated, ${ }^{2}$ there is no generally available, integrated set of computational software for developing and capturing materials information to make it available for developing new theoretical understandings or for use in engineering design.

The Materials Genome Initiative (MGI) established that the acceleration of the process of materials discovery and development is a major national need, and identified the development of open-source software and a materials information infrastructure as important goals of MGI. ${ }^{2}$ Development of community-based open-source, computationally efficient and integrated codes is a major opportunity for rapid advancement of computational materials science and engineering and is a primary focus of the Center for Predictive Integrated Structural Materials Science (PRISMS Center) described in this article. The PRISMS Center was established in 2012 and is a multi-year, multi-investigator activity funded by the U.S. Department of Energy Basic Energy Science flagship MGI program "predictive theory and modeling".

The overarching goal of the PRISMS Center is the establishment of a unique scientific framework and associated integrated open-source software to enable accelerated predictive materials science. The PRISMS Center has three key thrusts and associated objectives:

- PRISMS Computational Software Develop and establish PRISMS Software, a suite of integrated, open-source multi-scale computational tools for predicting the microstructural evolution and mechanical behavior of structural metals.

- The Materials Commons Develop and deploy the Materials Commons, a virtual collaboration space and repository for curating, archiving and disseminating information from experiments and computations.

- PRISMS Integrated Science Use Cases Significantly advance the science of metals by integrating the use of PRISMS computational tools with advanced experimental methods focused on specific scientific challenges. The use cases described here demonstrate the application of the integrated PRISMS capability for making major advances in the quantitative and predictive understanding of microstructural evolution and mechanical behavior of metals and alloys.

The architecture of the PRISMS integrated framework is shown in Fig. 1. This framework, and associated computational tools and protocols, were designed to become a widely adopted, core scientific methodology that forms a common basis for the rapid development of new materials science and insertion into the growing field of Materials By Design/Integrated Computational Materials Engineering (ICME). ${ }^{2-5}$ This framework builds on the substantial efforts of previous researchers in the area in ICME, multi-scale modeling and materials information e.g., Refs. 3-9, the review of which is beyond the scope of this article but which have been reviewed elsewhere e.g., Refs. 10 and 11. The PRISMS framework is substantively different than these preceding efforts in that it incorporates an integrated suite of open-source software and a materials information repository to make this framework accessible and usable by the broad materials community. We envision this as providing a strong, uniform basis for accelerating the development of next-generation ICME capabilities. While other open-source codes are available in targeted areas e.g., Refs. 12-17, they have not been integrated with other tools as accomplished here.

Magnesium ( $\mathrm{Mg}$ ) and its alloys serve as a prototype materials system for demonstrating the capability of the PRISMS framework and the scientific advances it enables. The behavior of $\mathrm{Mg}$ alloys presents a wide array of unsolved scientific challenges, including complex precipitation reactions, dislocation interactions in this hexagonal closepacked (HCP) system, twin formation and growth and the influence these deformation modes have on tensile and fatigue behavior. Compared to other structural metals, the development of commercial $\mathrm{Mg}$ alloys and our understanding of $\mathrm{Mg}$ alloy physical metallurgy is less mature. While alloys of aluminum and nickel can contain five to ten alloying elements, $\mathrm{Mg}$ alloys have remained simple and are typically based on only two or three alloying additions. Mg alloys also have significant potential for reducing the weight of automobiles and other transportation systems, and thus for improving fuel economy and reducing emissions. Many of these potential applications require significant improvements in strength, fatigue and corrosion resistance. Adoption of an ICME or Materials by Design approach for rapid development and insertion of new $\mathrm{Mg}$ alloys into the transportation industry is a major MGI opportunity. Developing a capability for enabling the rapid assessment of such advanced alloys is a central focus of the PRISMS Center.

This paper provides an overview of the PRISMS Center progress and future directions. For additional information we refer the reader to our published papers and the Center website: http://pri sms-center.org/.

\section{PRISMS Open-Source Integrated Software}

Currently, four primary codes make up the PRISMS open-source integrated software: CASM, PRISMS-PF, PRISMS-Plasticity and DFT-FE. Three of these codes (CASM, PRISMS-PF, and 


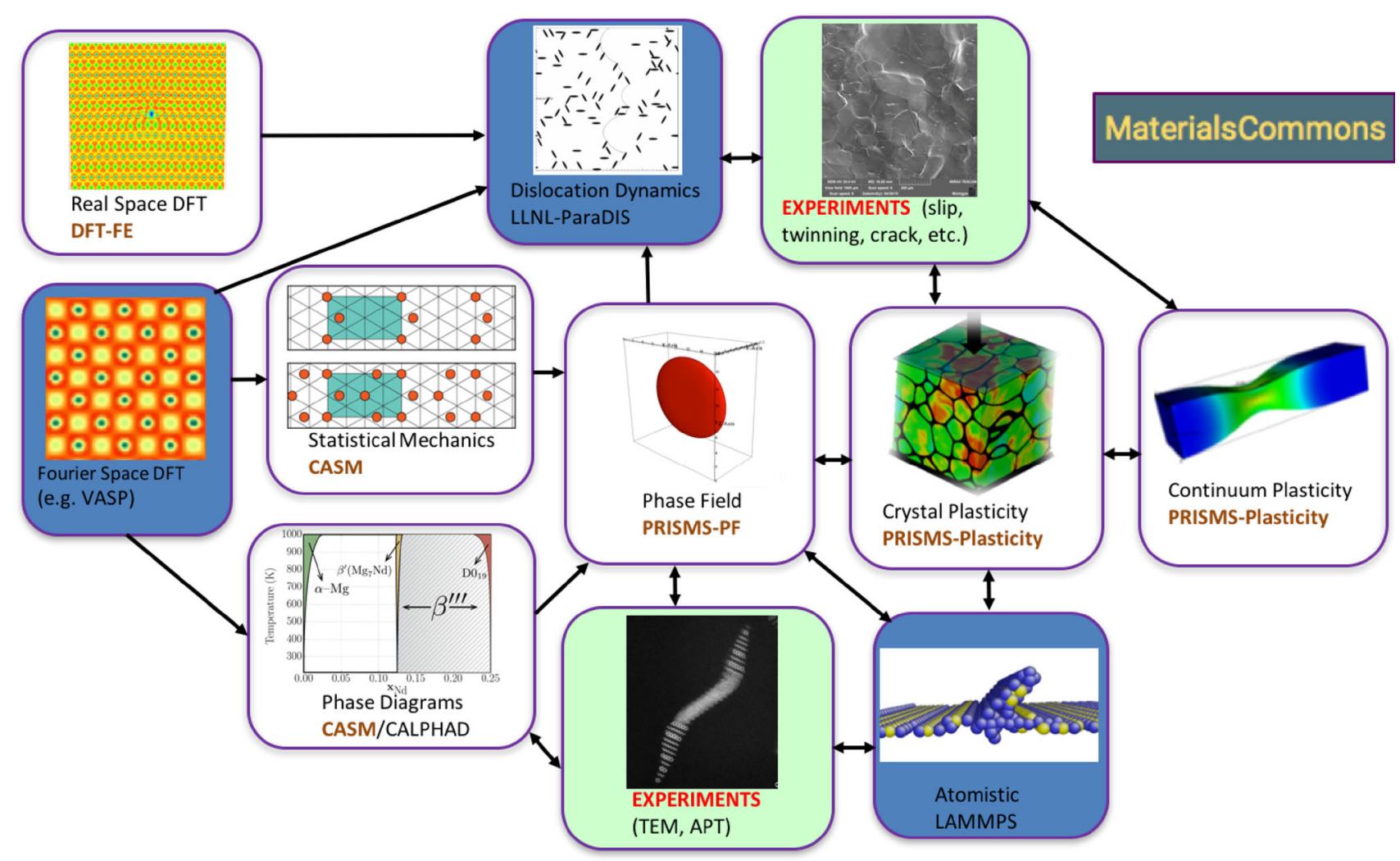

Fig. 1. The PRISMS Integrated Framework. White PRISMS Software, Green PRISMS Experiments, Blue Existing External Software.

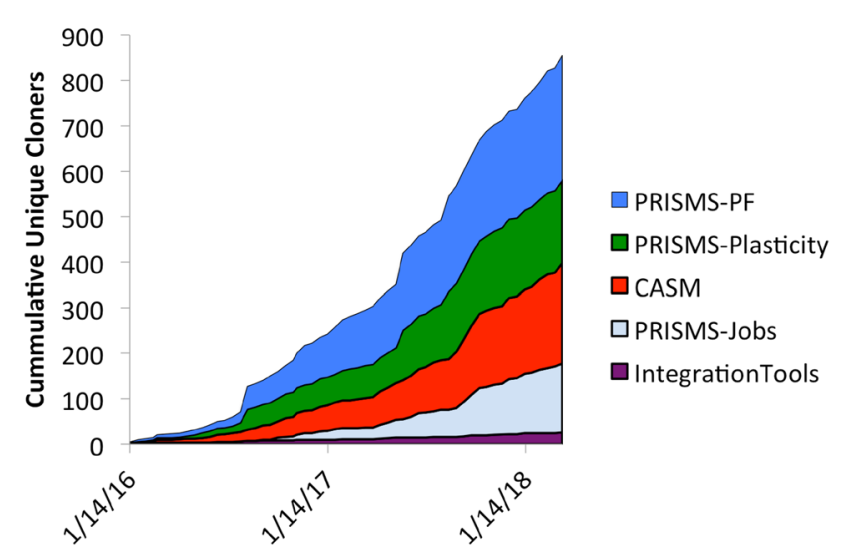

Fig. 2. Cumulative unique clones of PRISMS codes since January 2016.

PRISMS-Plasticity) were released in 2015 on GitHub, and major updates have been released annually since. These codes are now considered to be stable and to significantly advance the state of the art. Where feasible, benchmarking, including use of standardized benchmark problems, ${ }^{18}$ has demonstrated that their computational efficiency is superior or equivalent to other open-source codes at similar levels of sophistication and capability. As of March 2018, the GitHub pages for these codes have been viewed over 48,000 times, and over 855 unique clones (copies) have been downloaded by users. Figure 2 shows the number of downloads over the past 3 years. The PRISMS user and code developer community is growing in the U.S., Europe, Asia and Australia. The PRISMS software is developed under the open-source LGPL license. Each software package includes a substantial user manual, commented source code and unit tests. As part of our annual workshop described below, we have trained more than 100 users over the past 3 years.

DFT-FE, the fourth of the primary PRISMS Software codes, was released in August 2018. It is an advanced, finite element-based real-space code for density functional theory (DFT) calculations. Finally, two sets of integration software, PRISMSIntegrationTools and PRISMS-Jobs, have been developed and released to handle interactions between PRISMS codes, Materials Commons, and computing clusters.

Three of the PRISMS codes (PRISMS-PF, PRISMS-Plasticity and DFT-FE) use the finite element method in their calculations and are built on the powerful and popular deal.II finite element library. ${ }^{19}$ The deal.II library provides adaptive refinement of distributed octree meshes, hybrid parallelism (combining MPI, threads, and vectorization) with demonstrated scaling for over 16,000 processors, spectral finite element basis functions of arbitrarily high order, and support for matrix-free calculations. The deal.II library supports a wide 
variety of quadrilateral (2D)/hexahedral (3D) element types, including standard Lagrangian elements and Gauss-Lobatto elements. PRISMS Software provides the materials community with a user-friendly interface for phase field, crystal plasticity, continuum plasticity and real space DFT simulations with the advanced capabilities of the deal.II library.

\section{CASM: A Clusters Approach to Statistical Mechanics (https://github.com/prisms-center/ CASMCode)}

The rational and directed design of new materials must invariably rely on a robust and self-consistent description of thermodynamic and kinetic properties within a given chemical composition design space. Crucial in such descriptions are free energies, as they are essential to establish phase stability. Free energies also encapsulate all other thermodynamic information about a system in the form of first and second derivatives, including chemical potential versus composition relationships, heat capacities, and elastic moduli. Kinetic theories, such as generalized phase field models, also rely on free energies along with kinetic coefficients of unit processes that include diffusion and interface migration to describe microstructure evolution and corrosion processes. A major goal of this effort is to connect the electronic structure of a material to its finite temperature thermodynamic and kinetic properties. The statistical mechanical approach being employed for multi-component crystalline phases relies on effective Hamiltonians to account for configurational and vibrational degrees of freedom (i.e., cluster expansion and lattice dynamical Hamiltonians). ${ }^{20-28}$ Effective Hamiltonians serve to extrapolate accurate first-principles electronic structure calculations within Monte Carlo simulations to enable the calculation of thermodynamic quantities.

To implement these methods, a completely new open-source version of the CASM software package $^{24,25,29}$ has been developed. CASM is designed to predict finite temperature phase stability of crystalline phases in alloys with an arbitrary number of components and arbitrary crystallographic complexity. Using group theory techniques from crystallography, CASM automates the construction of an effective Hamiltonian for a particular parent crystal structure and spawns off first-principles electronic structure calculations needed to parameterize the interaction coefficients of the effective Hamiltonian. Once a Hamiltonian has been constructed, CASM subsequently generates customized and highly optimized code to evaluate the Hamiltonian for that particular system in canonical and grand canonical Monte Carlo calculations. The software can be applied to any periodic crystal in three dimensions (bulk crystals), two dimensions (special interfaces and grain boundaries) and one dimension. CASM provides wrappers to manage high-throughput firstprinciples calculations using both VASP ${ }^{30-33}$ and Quantum ESPRESSO, ${ }^{1,34}$ and enables the use of state-of-the-art methods for estimation of the expansion coefficients, including those based on genetic algorithms, ${ }^{35}$ compressive sensing, ${ }^{36}$ and depthfirst-search, ${ }^{29}$ via the popular machine-learning software packages Scikit-learn ${ }^{37}$ and DEAP. ${ }^{38}$

The open-source version of CASM is in active development and future releases are planned to enable generation of strain energy expansions in terms of symmetry-adapted strain order parameters $^{39}$ symmetry-adapted configuration order parameters, and compositional and configurational order parameter-dependent free energies, ${ }^{40}$ anharmonic lattice dynamical Hamiltonians, ${ }^{24,39}$ and prediction of multi-component diffusion coefficients in non-dilute solids ${ }^{41-49}$ with local cluster expansions $^{41-43}$ and kinetic Monte Carlo simulations, including accelerated methods. ${ }^{50}$

\section{PRISMS-PF: Phase Field Code (https:/github. com/prisms-center/phaseField)}

PRISMS-PF ${ }^{51}$ is a general-purpose open-source framework for phase field modeling using the finite element method. It has 22 pre-built applications, covering common uses of phase field modeling from spinodal decomposition to dendritic solidification (see Fig. 3). PRISMS-PF includes a state-of-the-art application for precipitate modeling used extensively in the Precipitate Evolution Use Case described below. ${ }^{52,53}$ This application features a KKS phase field model coupling to linear elasticity, including heterogeneous, anisotropic elastic constants and composition/order parameter-dependent misfit strains. It also supports separate order parameters for different precipitate variants. All PRISMS-PF applications have access to its nucleation capabilities, which leverage the order-parameter-only explicit seeding approach, ${ }^{54,55}$ allowing an implementation of a user-specified nucleation probability density model (e.g., classical nucleation theory) to stochastically insert nuclei.

PRISMS-PF is divided into three components: the applications, the core library, and the test suite. Most users only need to interact with the applications component, often through a parameters file where numerical and model constants are set. Users may also customize the governing equations, initial conditions, and post-processing expressions. The core library component handles functionality shared between all applications including the finite element calculations as well as input-file parsing, mesh creation, and output of results. Finally, the test suite component includes unit tests, regression tests, and analytic solution comparisons for code verification.

In addition to provide a user-friendly and flexible framework for phase field modeling, the computational performance of PRISMS-PF exceeds that of 

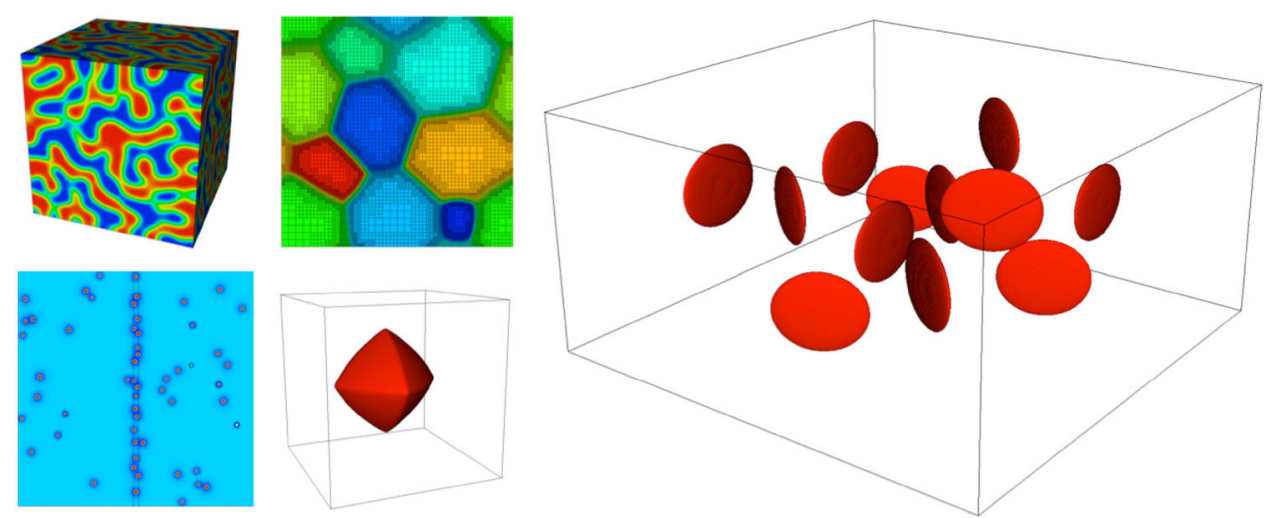

Fig. 3. Examples of simulations conducted using PRISMS-PF: spinodal decomposition, grain evolution, nucleation on a grain boundary, a particle with strong anisotropy, and evolution of multiple precipitates.

standard approaches. This performance is enabled by five features from the deal.II finite element library $^{19}$ upon which it is built. First, it is parallelized on three levels to fully take advantage of modern computer architectures: inter-node level (MPI communication), intra-node level (Thread Building Blocks), and intra-core level (vectorization up to AVX512). Near ideal scaling with PRISMS-PF has been demonstrated for more than 1000 cores. Second, adaptive meshing capabilities ${ }^{56}$ reduce the resolution of the mesh away from interfaces where the variables are nearly constant. The increase in speed from adaptive meshing is highly dependent on the problem being solved, from 20 times $^{52}$ to multiple orders of magnitude. ${ }^{57}$ Third, the deal.II library's state-of-the-art sum-factorization features $^{58}$ eliminate the need for the creation of a global sparse matrix. Instead, operators are applied cell-by-cell, reducing the time spent accessing memory. For quadratic elements, this approach has been shown to be $4 \times$ faster than the traditional approach. $^{51}$ Fourth, the use of Gauss-Lobatto quadrature enables low-cost explicit time stepping without the need for "mass lumping" or a matrix inversion by yielding a diagonal (and thus trivially invertible) mass matrix. ${ }^{58}$ Fifth, PRISMS-PF offers choices of quadratic or cubic elements, in addition to linear elements. The higher-order elements have improved error convergence and use fewer floatingpoint operations per degree of freedom than linear elements, as the result of the sum-factorization approach. $^{58}$

Even without the activation of adaptive meshing, the other four performance-enhancing aspects of PRISMS-PF enable it to be twice as fast as a standard single-purpose MPI-parallelized finite difference code. ${ }^{51}$ With adaptive meshing activated, but under conditions unfavorable for adaptive meshing, PRISMS-PF is still seven times faster than a finite difference code.

Initially released in 2015, PRISMS-PF remains under active development, with new major features released annually. New planned features to be released in 2018-2019 include a new flagship corrosion application, a grain re-assignment algorithm for grain growth simulations, implicit time stepping with adaptive time step control, and a GUI using the Rappture Toolkit. ${ }^{59}$

\section{PRISMS-Plasticity: Continuum and Crystal Plasticity Finite Element (CPFE) Code (https://www.github.com/prisms-center/ plasticity)}

The PRISMS-Plasticity code is a parallel numerical framework for implementing continuum and crystal plasticity models. It is developed on a base framework for parallel implementation of elliptic boundary value problems using the deal.II opensource finite element library. For crystal plasticity, we have implemented HCP, BCC and FCC single crystal elasto-plastic constitutive models. For continuum plasticity, von Mises plasticity with J2-flow theory, isotropic and kinematic hardening are included. The current implementation is for quasistatic conditions; however, deal.II allows both dynamic and explicit implementations which may be the subject of future developments by the PRISMS Center or external open-source collaborators. Using experimental or synthetic 3D polycrystalline representative volume elements (RVE) as input, the crystal plasticity finite element (CPFE) code can compute the anisotropic response of crystalline aggregates to large-strain quasi-static mechanical loading in the form of grain-level stress contours and pole figures. The material deformation is solved based on a constitutive response driven by mechanisms of dislocation slip, twinning and crystal reorientation. The evolution laws for micro-scale state variables, namely, slip and twin system resistances are based on previous work. ${ }^{60-63}$ Twin reorientation is tracked using the predominant twin reorientation (PTR) scheme ${ }^{64}$ at the level of individual elements. The code has the capability to read in microstructures created by DREAM3D and NEPER, popular packages for microstructure 
generation, and supports both structured and unstructured grids. The parallel code output can be imported by software such as ParaView for visualization and to generate movies. The code output can be read in by PRISMS Center-provided scripts or by external codes for post-processing to pole figures and orientation distribution functions. Additional capabilities include handling of twophase materials and a back-stress implementation for modeling cyclic loading (Fig. 4).

Code efficiency has been significantly improved using a new iterative procedure to compute active slip systems that ensures all slip systems lie on or within the single crystal yield surface. The algorithm also includes adaptive time stepping to speed up calculations and has allowed significant improvements in convergence in non-linear finite element iterations. PRISMS-Plasticity has been demonstrated on large-scale problems with millions of degrees of freedom running on thousands of processors. An example of such simulations is shown in Fig. 4, which took only 96 CPU-h. The microstructural strain response predicted by the PRISMSPlasticity CPFE module has been validated by comparison with strain maps from SEM-DIC measurements, and it provides a reliable indicator of stress heterogeneity within grains as well as Schmid factor variations due to the effect of neighbor grains. ${ }^{65,66}$ The crystal plasticity code has been used for computation of dislocation density distribution for use in static recrystallization models ${ }^{67}$ and for initializing the single crystal parameters of other alloys ( $\mathrm{Ti}, \mathrm{Al}$, $\mathrm{Fe})^{68-70}$ in projects outside the PRISMS Center.

New features under development for PRISMSPlasticity include advanced capabilities such as gradient plasticity and 3D fracture simulation in additional to upgraded models for twinning and grain size effects.

\section{DFT-FE: Finite Element-Based Real-Space DFT (https://github.com/dftfeDevelopers/dftfe)}

Defects play a crucial role in influencing the mechanical response of crystalline solids, and examples include dislocations, solute impurities, precipitates, and grain boundaries. These defects, even when present in very small concentrations (a few parts per million), produce a significant macroscopic effect on the materials behavior through the longranged elastic fields that they generate. The strength and nature of these fields, as well as other critical aspects of the defect-core, are all determined by the electronic structure of the material at the quantum-mechanical length-scale. Hence, there is a wide range of interacting length-scales, from electronic structure to continuum, that need to be resolved to accurately describe defects in materials and their influence on the macroscopic properties of materials.

Building on prior work, we have developed a framework for conducting large-scale real-space electronic structure calculations using Kohn-Sham density functional theory $\left(\right.$ KSDFT) ${ }^{71-73}$ (cf. Figure 5). Specifically, the capabilities developed in the DFT-FE code have been aimed at enabling studies of extended defects in materials that have been out of reach using existing state-of-the-art DFT codes using a plane-wave discretization. In particular, the developed capabilities have three key distinguishing features: (1) it is based on a realspace formulation and a finite-element discretization $^{71}$ that allows for the consideration of general geometries and boundary conditions, which is important in studying extended defects such as dislocations whose geometry is not compatible with periodic boundary conditions; (2) the cubic scaling bottleneck of conventional KSDFT calculation is overcome by the development of reduced-order scaling methods, ${ }^{72}$ which are applicable to both insulating and metallic systems, and have enabled large-scale KSDFT pseudopotential calculations on systems containing $\sim 10,000$ atoms; and (3) the code has been demonstrated to scale well on parallel architectures, with over $90 \%$ efficiency on many thousands of processors. Open-source software based on some of these capabilities has been developed with the first public release of the beta version recently announced in August 2018. This release features the following capabilities: (1) periodic and non-periodic calculations using norm-conserving Troullier Martins and ONCV pseudopotentials and density-based exchange correlation functionals

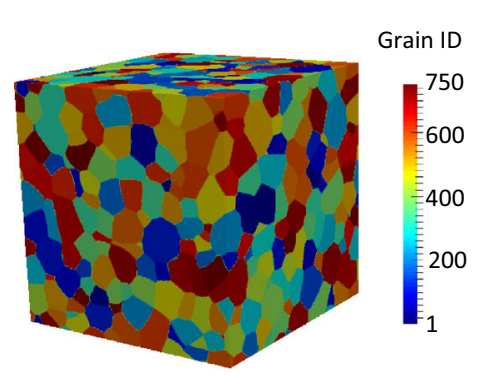

750 grains, $600 \times 600 \times 600 \mu \mathrm{m}^{3}$
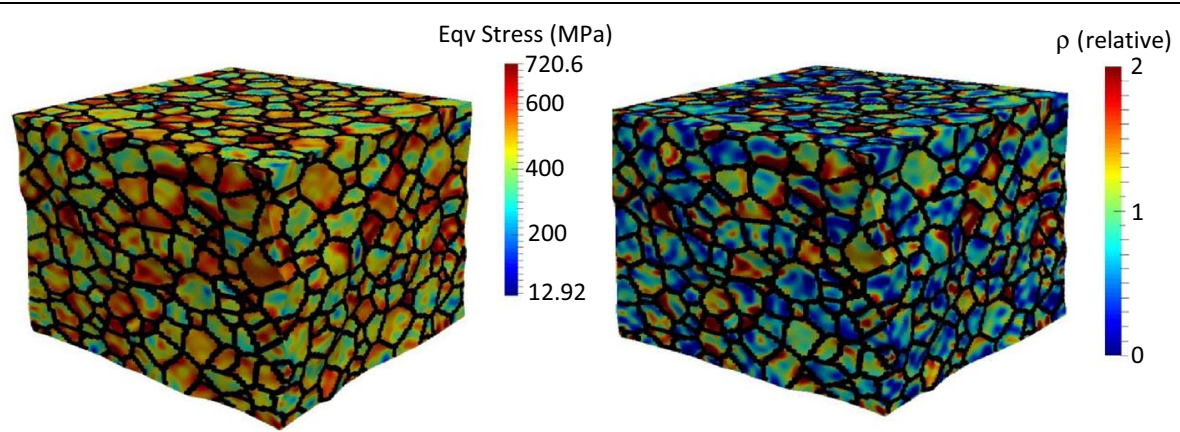

Parallel CPFE simulation: results for stress (left) and relative dislocation density (right)

Fig. 4. PRISMS-Plasticity CPFE simulation of deformation of a polycrystalline sample for use with a Mg recrystallization model. 


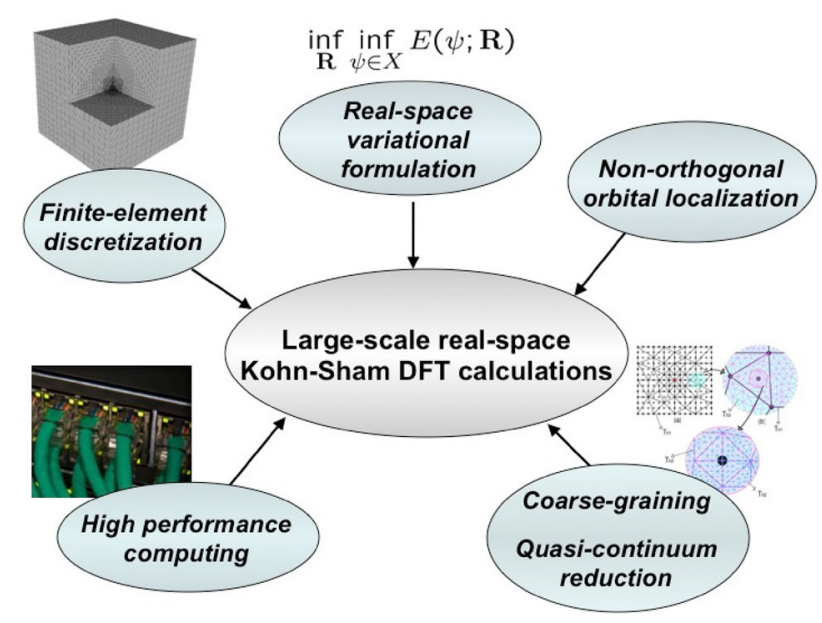

Fig. 5. Primary aspects of the development of large-scale realspace Kohn-Sham DFT calculations to enable electronic structure studies on extended defects.

(LDA/GGA); (2) mesh-adaption capability based on p4est (an efficient implementation of a parallel octree data structure); and (3) computation of ground-state energy including geometry optimization.

\section{PRISMS-Integration Tools}

Integration of the PRISMS Software codes with each other, Materials Commons and external codes is an important aspect of the PRISMS framework. To accomplish this, the Integration Tools package was implemented as a generic interface for passing parameters into and out of the PRISMS codes, decoupling the mathematical and numerical representation of the parameters and their derivatives from their use. As an example, using Integration Tools, the PRISMS-PF code is written to use a free energy function and its derivatives, without knowing the specific form parameterized by CASM. The current version includes support for passing arbitrary analytical functions, including piece-wise functions, series expansions, and tensor products of series expansion for multivariate functions, and linear finite element interpolations.

\section{Advanced Research Methods}

In addition to DFT-FE, which has become a mainstream PRISMS-supported code, we have pursued other advanced models for simulating phase evolution and fracture. These include mechanoChem, phase field crystal developments and variational multi-scale models for fracture. ${ }^{74}$

mechanoChem is a suite of codes for continuumscale models of coupled, multi-physics phenomena governed by the partial differential. This code suite (v0.1) was publicly released on GitHub in September 2016, and is now under continuous integration and development by the Garikipati Group. These models are applied to initial and boundary value problems involving structural alloys, energy storage materials and other systems of current and future interest $^{75-81}$ at length scales of $\sim 100 \mathrm{~nm}$ to $\sim 1$ $\mathrm{cm}$. The mechanoChem models and codes have been developed to take as inputs quantities such as free energy densities, elasticity tensors, reaction rates, and mobility and conductivity tensors. These quantities are computed by combining methods that operate at smaller length and time scales. The free energy densities can be computed by DFT followed by cluster expansions, the elasticity tensors by DFT, and the reaction rates, mobilities and conductivities by DFT, cluster expansions and statistical mechanics methods such as kinetic Monte Carlo.

mechanoChem is a C++ library of Isogeometric Analysis (IGA) and Finite Element Method (FEM) codes to solve coupled, initial and boundary value problems of reaction-transport, elasticity, heat conduction and electrostatics. It is built on well-established, open-source parallel software libraries including deal.II, PETSc (parallel data structures, solvers and preconditioners), Trilinos (automatic differentiation, solvers and preconditioners) and PetIGA (IGA toolkit). mechanoChem also employs a hybrid parallel programming model of MPI + OpenMP + Vectorization, well suited for memory-limited multi-core architectures. In addition to the native capabilities of these libraries, mechanoChem also can interface with other codes, such as Gmsh and CUBIT for mesh generation, and is itself easily called by Python scripts in more complex workflows.

The phase field crystal (PFC) model is an approach that can simulate crystalline materials with atomistic resolution but at a diffusive timescale. ${ }^{82}$ Within the PRISMS Center's Advanced Methodology efforts, a PFC model has been developed for HCP crystals ${ }^{83}$ based on the structural PFC model. ${ }^{84,85}$ We have shown that prior models did not possess correct lattice parameter ratios for the HCP structure. Using the new model, the grain boundary energy was calculated as a function of the misorientation angle. The results were compared to those obtained using molecular dynamics, ${ }^{86}$ which showed agreement of relative grain boundary energies within model uncertainty. In addition, through two-dimensional simulations, we have shown that PFC results better match the grain boundary structures and misorientation dependence of grain boundary energies obtained by molecular dynamics at high temperatures (70-80\% of melting temperature) as compared to lower temperatures. ${ }^{87}$

\section{Materials Commons (https://materialscommons.org)}

A critical challenge for the materials community continues to be the integration of information from a wide variety of sources into knowledge repositories that are accessible by the broad technical 
community. Such knowledge repositories are essential to disseminate, validate and archive the results of scientific investigations, and, importantly, to collaborate and expand our scientific horizons. Fields as far ranging as biology, astronomy, climate change and earthquake science have embraced this concept. However, the materials field has lagged behind, and this limits ready access to the latest results of scientific endeavors, including details of experiments and models. ${ }^{5,11}$ It also leads to the costly and time-consuming development of redundant information. One of the major goals of the MGI is the establishment of a materials information infrastructure for sharing materials information. ${ }^{3}$

The Materials Commons is a collaboration platform and information repository for the materials community. ${ }^{88}$ It aims to be a seamless part of the scientific workflow and has been designed to cover the entire data lifecycle, from data acquisition and sharing through analysis and re-use. It provides a common site for researchers to store, share, curate, and re-use experimental and computational data, with a data model designed for the needs of the materials community. To accommodate different needs and use cases across the data lifecycle, the Materials Commons consists of a website, materialscommons.org, tailored for data sharing, discovery, organization, and collaboration, and a Python API and command line tool ${ }^{89}$ for data capture, metadata creation, and data analysis.

Researchers using the Materials Commons start by creating a project that is shared with their collaborators. Using the website or Python API and command line tools, anyone granted access to the project can upload files to be shared amongst collaborators. Importantly, associated metadata, namely key data describing the scientific workflow and observed structure-processing-property relationships is stored in the database. An example of the electronic laboratory notebook representing an experimental workflow is provided in Fig. 6. Process templates describing what data are captured for each type of process (a particular experimental or computational test or analysis) provide data standardization and can be created via the website. These data can be automatically generated by parsing spreadsheets that have been annotated to allow Materials Commons to understand them, or by using the Python API to develop integrations with software packages or and analysis scripts. Once data are uploaded to the project, all collaborators can visualize the workflow on the website and output spreadsheets or use the Python API to query data for analysis. When ready, the entire project or a subset can be easily selected to be packaged into a dataset that is then given a DOI and made available to the wider community for download and re-use.

PRISMS Center members have been using the Materials Commons since 2014. The Materials Commons was made available to the broader technical community in August 2016, and since that time over 180 users have been registered. Over 1.1 million files have been uploaded in 150 sets of data. All components of the Materials Commons are available open-source. ${ }^{90}$ The Materials Commons is one of several efforts to develop data services and analysis services ${ }^{91-93}$ sponsored by MGI. A growing activity within this community is the development of an integrated, interoperable materials information infrastructure. ${ }^{11,88,91,93}$

\section{Use Cases}

A unique aspect of the PRISMS Center is our focus on quantitative prediction of alloying and processing effects on microstructures linked to properties. Establishing this predictive linkage is essential for accelerating the design and discovery of new materials and an important priority for the Center. The Center is organized around Use Case topic areas that serve as a central forum for scientific collaboration and for tightly coupling experimental activities with integrated multi-scale modeling. These Use Cases are meant to serve as demonstrations and test beds for how PRISMS Software, systematic experiments and Materials Commons can be used by others to rapidly advance science in structural metals. These Use Cases offer a means to partition the overall PRISMS framework into tractable problems addressing particular scientific, modeling and experimental challenges. We have made significant progress on three Use Cases: namely, Precipitate Evolution, Tensile Behavior and Fatigue Behavior of advanced magnesium alloys as described below. The Use Cases are designed to integrate and operate together. For example, results of the Precipitate Evolution Use Case are being passed to the Tensile Use Case for use in predicting the influence of precipitate variables on strength.

Magnesium-Rare Earth alloys exhibit exceptional strength properties; ${ }^{94-102}$ however, they have been largely developed by traditional Edisonian techniques. We have thus focused our Use Case activities on the Mg-Nd-Y alloy system as an exemplar for the acceleration in fundamental understanding that can occur by tightly coupling experiments, theory and integrated multi-scale modeling.

\section{Precipitate Evolution Use Case}

The objective of the Precipitate Evolution Use Case is to provide insight into the nano-scale precipitates that strengthen many alloys. DFT, statistical mechanical, phase field, and dislocation dynamics calculations were combined with quantitative $2 \mathrm{D}$ and $3 \mathrm{D}$ experimental characterization to demonstrate an integrated platform that can predict precipitate microstructure and its influence on strength. This demonstration centers on the Mgrare earth (Mg-RE) family of alloys, which have garnered increasing interest for structural 


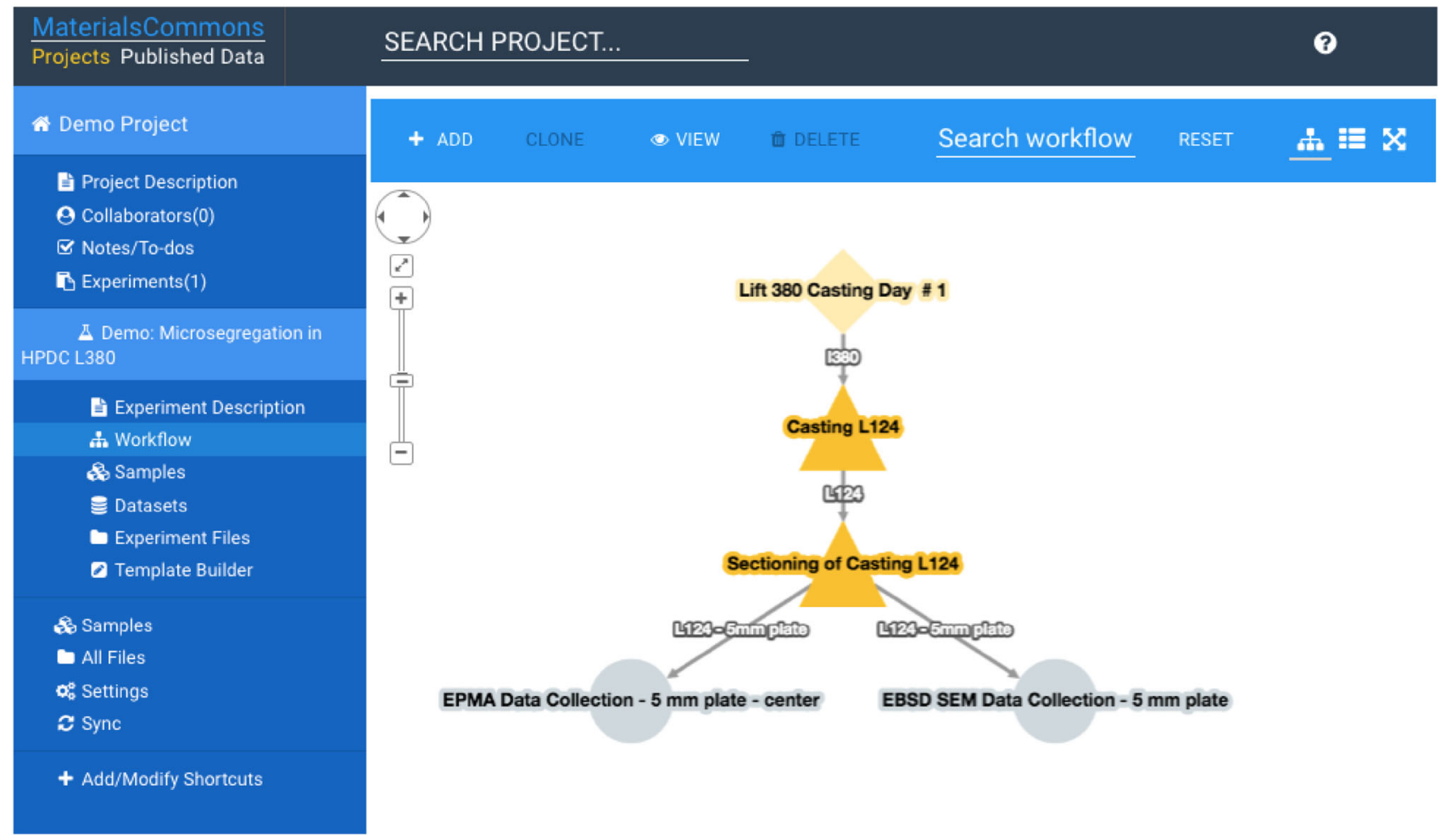

Fig. 6. Screenshot of the visualization of workflow for a demonstration project in the Materials Commons. Nodes represent actions that have been performed in a scientific workflow.

components in automotive, aerospace, and portable consumer device applications. ${ }^{102}$ Optimal strength in these alloys is achieved by a complex and previously poorly understood precipitation sequence. ${ }^{100,101}$ It involves the nucleation of Guinier-Preston (GP) zones followed by the precipitation of metastable nano-scale HCP-based phases (historically referred to as $\beta$ '). Further aging leads to the formation of BCC-based precipitates, referred to as $\beta_{1}$, that form from and coexist with the original $\beta$, precipitates. Since these precipitates are metastable and appear only in relatively new alloy systems, their thermodynamic and kinetic properties are not included in commercial thermodynamic databases.

As part of this Use Case, a combined firstprinciples and experimental study ${ }^{103}$ of precipitation in supersaturated solid solutions of Mg-rich $\mathrm{Mg}-\mathrm{Nd}$ alloys was undertaken to establish thermodynamic driving forces for the formation of GP zones and precipitates. The CASM software package was used to calculate a first-principles-based metastable HCP temperature-composition phase diagram (Fig. 7a) using a cluster expansion Hamiltonian and Monte Carlo calculations. Aging studies were performed in parallel on dilute $\mathrm{Mg}-\mathrm{Nd}$ alloys which were then characterized with HAADF-STEM, an example of which is shown in Fig. 7b. This joint study has revealed that the early-stage decomposition of these alloys is dominated by the thermodynamic metastability of a unique hierarchy of Nd orderings on the HCP crystal structure, which we label $\beta$ "'. These $\beta$ "' orderings have a higher $\mathrm{Nd}$ composition than $\beta$ 'ordering and have linear strips of Nd-hexagons at non-periodic intervals (as seen in Fig. 7b). This investigation demonstrated that precipitates with this $\beta$ "' ordering appear during aging between the formation of GP zones and the formation of $\beta_{1}$ precipitates, rather than $\beta^{\prime}$ and $\beta^{\prime \prime}$ orderings as was previously believed. ${ }^{104}$

This in-depth investigation of the $\mathrm{Mg}-\mathrm{Nd}$ binary alloy laid the groundwork for high-throughput firstprinciples studies of ordering tendencies in all firstrow Mg-RE binary alloys, ${ }^{105}$ which provide new and important directions for future alloy developments. As described in Ref. 105, an ordering labeled $\beta_{\mathrm{S}}$ ' is predicted to form in the $\mathrm{Mg}$ - $\{\mathrm{Sc}, \mathrm{Y}, \mathrm{Tb}, \mathrm{Dy}$,$\mathrm{Ho}, \mathrm{Er}, \mathrm{Tm}, \mathrm{Lu}\}$ binary alloys, while an ordering labeled $\beta_{\mathrm{P}}$ "' is predicted to form in the Mg$\{\mathrm{La}, \mathrm{Ce}, \mathrm{Pr}, \mathrm{Nd}, \mathrm{Pm}, \mathrm{Sm}\}$ binary alloys. For $\mathrm{Mg}-\mathrm{Gd}$, the two orderings were approximately equally favorable. These calculations are consistent with the observations for the four well-characterized binary alloys, Mg- $\{\mathrm{Nd}, \mathrm{Sm}, \mathrm{Y}, \mathrm{Gd}\}$. The predictions for the remaining 11 binaries could be tested with future experiments. The coherency strains for the two types of orderings are hypothesized to determine the precipitate morphology, with $\beta_{\mathrm{S}}$ ' leading to rod-like precipitates (as in Mg-Y) and with $\beta_{\mathrm{P}}$ "” leading to lenticular precipitates (as in $\mathrm{Mg}-\mathrm{Nd}$ ). 

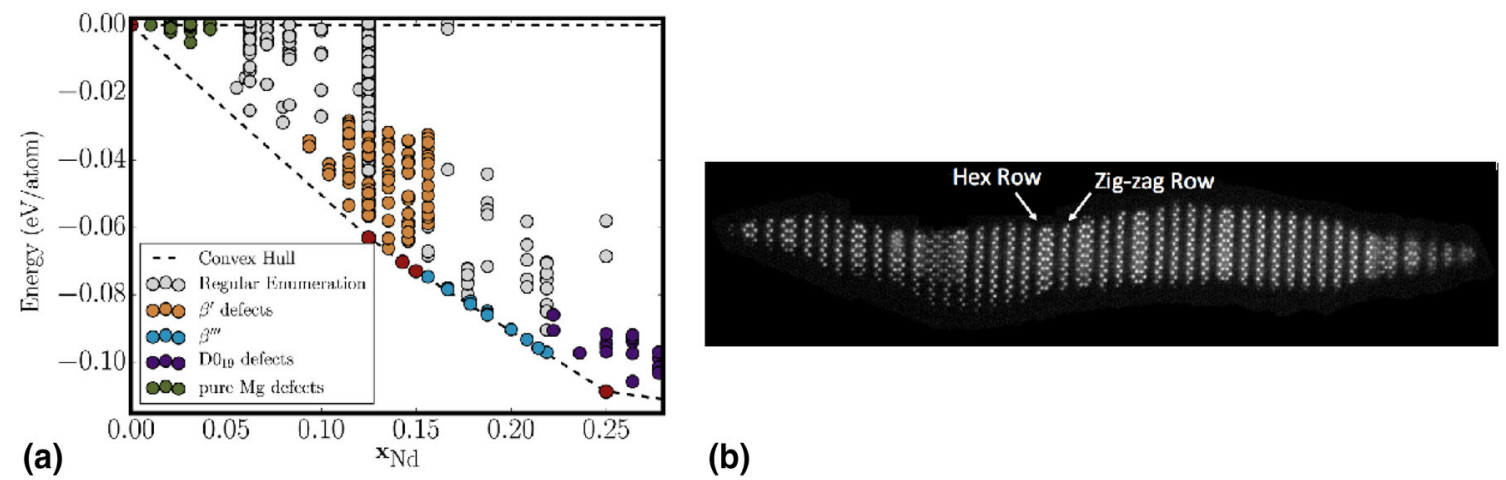

Fig. 7. (a) Formation energy of HCP structures in Mg-Nd calculated by DFT, showing the stability of a series of $\beta$ "' orderings. (b) HAADF-STEM image of a precipitate with the $\beta$ "' ordering depicting the combination of hex row and zig-zag rows. (Reproduced with permission from Ref. 103. Copyright 2016 Elsevier.

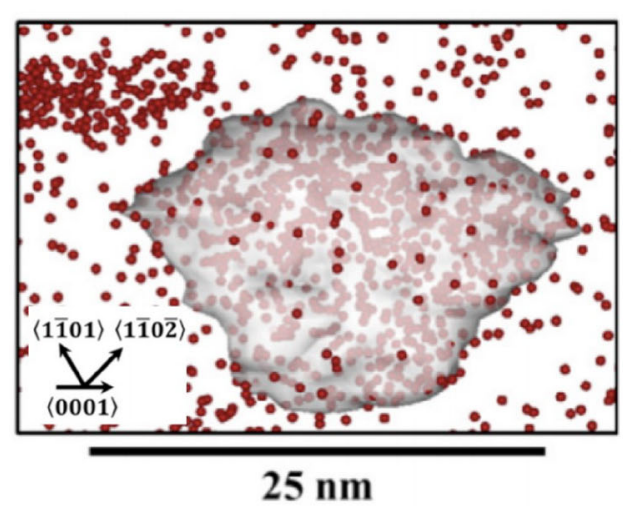

(a)

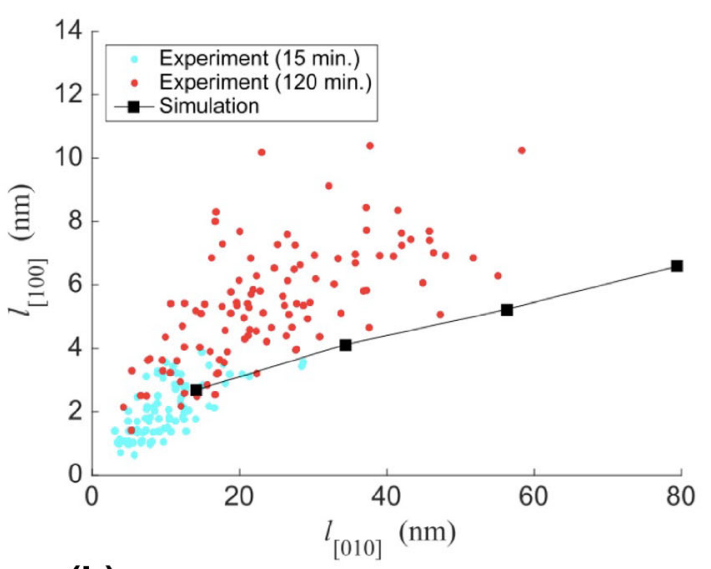

(b)

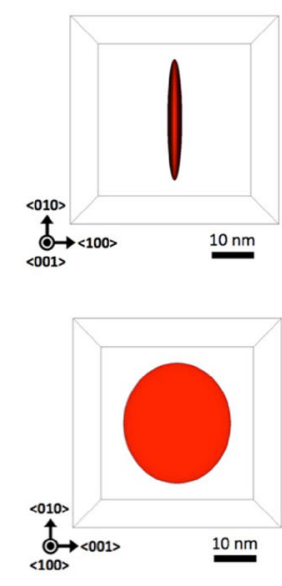

(c)

Fig. 8. (a) Atom probe tomography reconstruction of a $\beta$ "' precipitate in an Mg-Nd alloy. (b) Measurements of the dimensions $\beta$ "' precipitates in an Mg-Nd alloy viewed along [001] from HAADF-STEM and phase field simulations. (c) Equilibrium $\beta$ "' precipitate morphology in an Mg-Nd alloy predicted by phase field simulations. (Reproduced with permission from Ref. 108 (a) and Ref. 52 (b), (c). Copyright 2017 Elsevier.

Related first-principles statistical mechanics calculations have predicted new martensitic phases in $\mathrm{Mg}$-Sc that may lead to shape-memory behavior. ${ }^{106}$

Following this work on phase stability in Mg-RE alloys, the precipitate composition, morphology, dimensions, and number density during aging were studied experimentally and computationally. In $\mathrm{Mg}$ $\mathrm{Nd}$, the shapes of GP zones and $\beta$ " precipitates were determined through atom probe tomography (APT), and the dimensions along two imaging directions of many $\beta$ " precipitates were measured with HAADFSTEM (Fig. 8a and b). ${ }^{107-109}$ Phase field simulations using PRISMS-PF and parameterized entirely from first-principles calculations yielded precipitates with a shape consistent with observations once the uncertainty in the misfit strains is taken into account $^{52}$ (Fig. 8b and c). The phase field simulations also reproduced the elevated $\mathrm{Nd}$ composition indicative of the $\beta$ "' ordering and confirmed the role of the large misfit strain in the stabilization of this ordering. A similar combined experimental and computational approach was undertaken to examine the $\beta_{\mathrm{S}}$ ' precipitates in $\mathrm{Mg}-\mathrm{Y},{ }^{110,111}$ and confirmed that the rod morphology can be largely attributed to the coherency strains predicted by CASM.

$\beta_{1}$ precipitates are another important strengthening phase in Mg-RE alloys. New PRISMS-PF nucleation model features were used to simulate the evolution of $\beta_{1}$ precipitates in an $\mathrm{Mg}-\mathrm{Nd}$ alloy through concurrent nucleation, growth, and coarsening $^{112}$ (Fig. 9), yielding results consistent with experiments. The results of these simulations are being mapped to dislocation dynamics simulations to serve as a linkage between the Precipitate Evolution Use Case and the Tensile Behavior Use Case as described in the next section.

\section{Tensile Behavior Use Case}

In the tensile behavior use case, the PRISMS Framework was extended to predict the influence of 


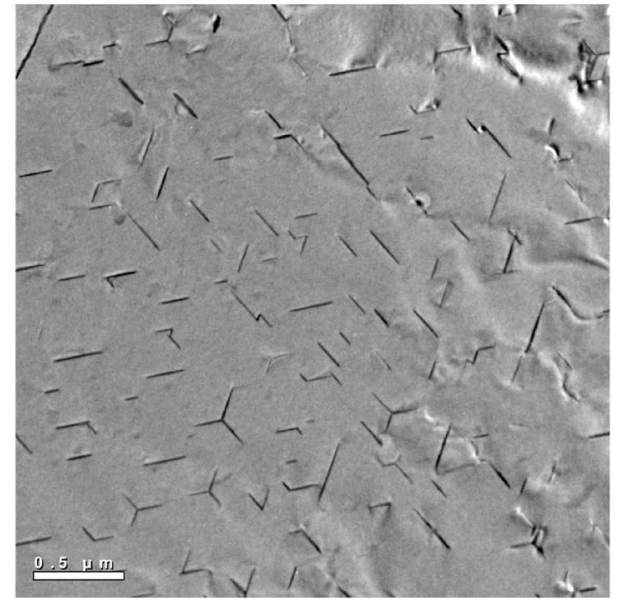

(a)

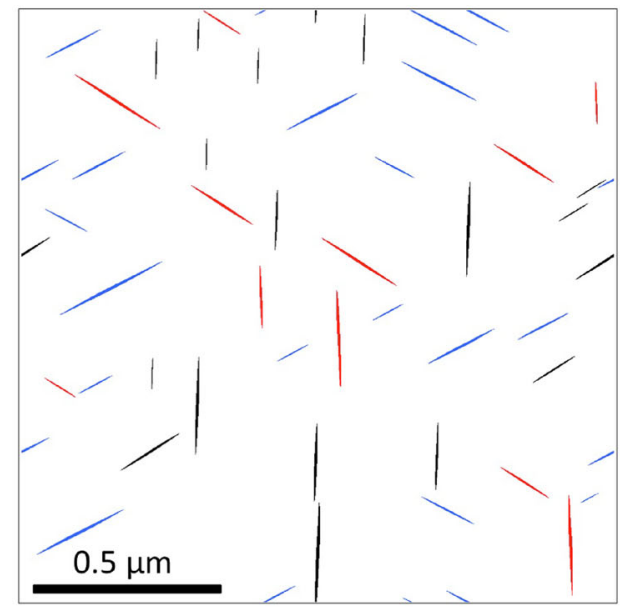

(b)

Fig. 9. (a) TEM image of $\beta_{1}$ precipitates in an Mg-Nd alloy after $9 \mathrm{~h}$ of aging. (b) Simulated microstructure using the phase field model for the same conditions where the color refers to pairs of orientation variants with the same stress-free transformation strain.

precipitate structure on yield strength and stressstrain response. This involves the integration of multi-scale models from atomistic simulations (Fourier and DFT-FE) with dislocation dynamics, crystal plasticity and continuum plasticity. This multi-scale modeling capability is integrated with unique experimental capabilities for measuring dislocation interactions with precipitates, grainlevel strain distributions (SEM-DIC) and carefully designed experiments for quantifying the influence of alloying, grain size and precipitate structure on stress-strain response. Real-space DFT calculations are being used to determine energetics of isolated dislocations in aluminum and magnesium. ${ }^{113,114} \mathrm{At}$ the dislocation-precipitate level, we are using the open-source code, ParaDiS, ${ }^{115}$ as the primary dislocation dynamics code to be integrated into the PRISMS Framework. A methodology has been developed to determine the critical resolved shear stress for dislocations moving on the basal slip plane through a given precipitate distribution. As an example, quantitative precipitate morphologies for an aged $\mathrm{Mg}-\mathrm{Al}-\mathrm{Zn}$ alloy were mapped to ParaDiS and the critical resolved shear stress determined. ${ }^{116}$ These results were combined with refined estimates of all other strengthening mechanisms and the computed estimates for yield strength were within $5 \%$ of the experimentally measured value. This demonstrated the feasibility of our integrated computational-experimental approach for predicting microstructural effects on properties.

Combining this approach with the results of the Precipitate Evolution Use Case, phase field simulations of $\beta_{1}$ precipitates in a $\mathrm{Mg}-\mathrm{Nd}$ alloy were mapped to ParaDiS input meshes. These were used in dislocation dynamics simulations (Fig. 10a) to predict the changes of the critical resolved shear stress throughout a simulated aging process. ${ }^{112}$ As shown in Fig. 10b, these results were informed by and validated using the results of an in situ TEM study of dislocation interactions with $\beta_{1}$ precipitates. ${ }^{117}$ Further, detailed in situ and ex situ experimental studies of dislocation-precipitate interactions for $\beta$ "' and $\beta_{1}$ precipitates in $\mathrm{Mg}$ $\mathrm{Nd}^{118,119}$ and $\beta$ ' in $\mathrm{Mg}-\mathrm{Y}^{109}$ elucidated new mechanisms governing the shearing of the precipitates that can improve future dislocation dynamics simulations. ${ }^{112,117}$

Results at the precipitate level were provided as inputs to the higher length scale represented by PRISMS-Plasticity. At this level, we have made extensive use of SEM-DIC ${ }^{120-122}$ to measure grain level strain maps. These strain maps allow us to gain insights into deformation mechanisms at the grain level and help in validation of the PRISMSPlasticity crystal plasticity modules ${ }^{65}$ In the latter, we compare strain distribution on a microstructure predicted by PRISMS-Plasticity with corresponding high-resolution SEM-DIC data, ${ }^{123}$ an example of which is shown in Fig. 11(top) for an advanced MgNd-Y alloy (WE43) compression test.

Using PRISMS-Plasticity (CPFE), tensile test data and an experimental EBSD (texture) map, we have reconstructed and tested representative polycrystals to develop crystal plasticity parameters for the WE43 alloy in two aging conditions (T5 and T6). This is similar to the approach used by Bhattacharyya et al. ${ }^{124}$ who have used integrated simulations to predict the influence of aging on flow stress and plastic anisotropy in WE43. In our current work, an interesting observation is that, in the $\mathrm{T} 6$ condition, pyramidal $<\mathrm{a}>$ is the least active mode, while in the T5 condition, pyramidal $<$ a $>$ appears to be a significant deformation mechanism (Fig. 11, bottom). This mechanism has been generally neglected in past studies on Mg-RE alloys. In addition, we can employ CPFE simulations to resolve traces of slip/twin systems that are close and cannot be readily distinguished. The integrated computational-experimental framework that has 


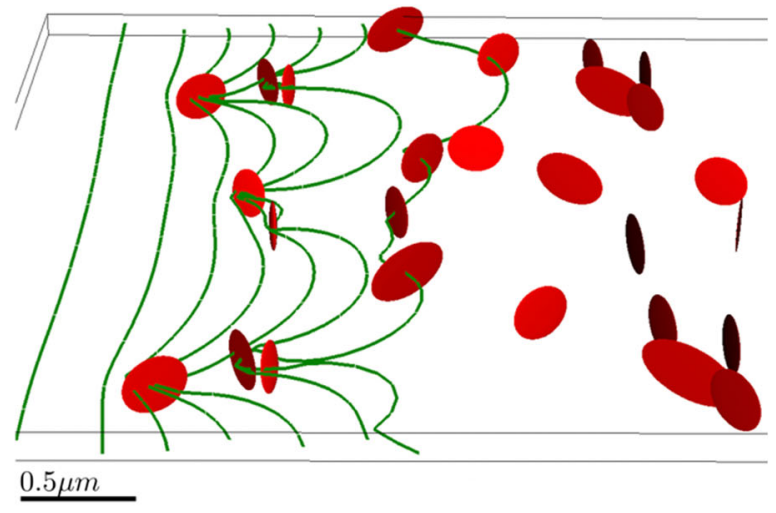

(a)

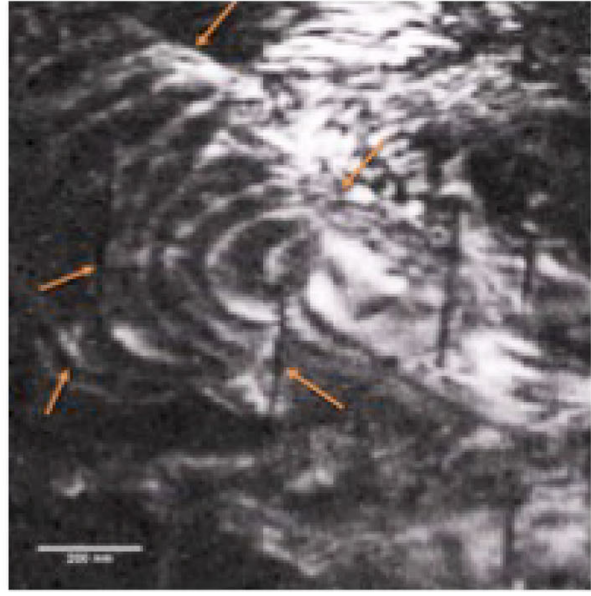

(b)

Fig. 10. (a) ParaDiS simulation of dislocation bowing at non-shearable precipitates in a Mg-Nd alloy and (b) in situ TEM image of dislocation bowing at precipitates (arrows) in an aged Mg-Nd alloy.
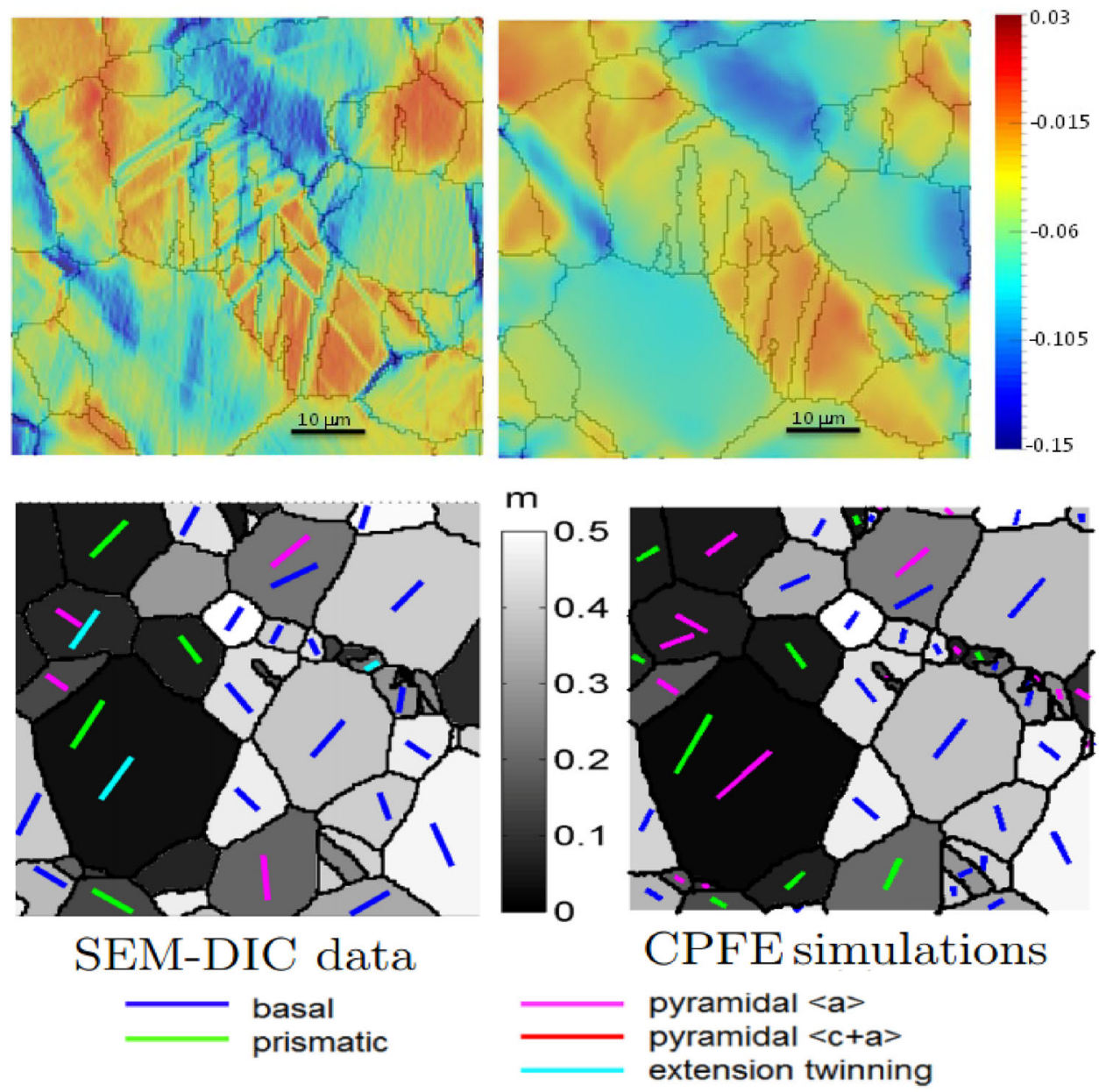

Fig. 11. (Top) Comparison of SEM-DIC measured strain map (left) and the CPFE simulation (right) for a compression test of Mg-WE43, Preexisting twins are also indicated. ${ }^{123}$ (Bottom) Comparison of slip traces from the SEM-DIC data against CPFE data for T5 alloy tension test. Top two predominant mechanisms from CPFE are plotted if two traces were seen in SEM-DIC. The background color is the basal Schmid factor. 
been established represents an important step in the direction of rapidly and accurately predicting alloying and microstructural effects on properties.

\section{Fatigue Behavior Use Case}

Similar to the tensile use case, the fatigue behavior use case involved the integration of multi-scale models including dislocation dynamics, crystal plasticity and continuum plasticity, with comprehensive experimental results used to quantify the influence of alloying, grain size and precipitate structure on cyclic stress-strain response, low cycle fatigue crack initiation and short fatigue crack growth.

The PRISMS Framework has been used to develop and demonstrate a capability for prediction of cyclic stress-strain response and low cycle fatigue behavior. Twinning is an important deformation mode in $\mathrm{Mg}$ and $\mathrm{Mg}$ alloys. In wrought material conditions containing strong crystallographic texture, twinning during cyclic compression loading followed by detwinning during cyclic tensile loading can lead to a strong Bauschinger effect that must be included in simulations. In this case, we have used synchrotron-generated high energy x-ray diffraction (HEXD) coupled with in situ cyclic loading to quantify the changes in twinning during cyclic deformation. ${ }^{125}$ An example of this behavior is shown in Fig. 12. At the early stages of cyclic deformation, twins were observed to form and completely detwin during the full loading cycle. As cycling continued, the volume fraction of twins increased as did the stresses at which twinning and detwinning occurred. At higher cycles, complete detwinning was no longer observed and residual twins remained and increased throughout the cyclic life. ${ }^{125}$

Accounting for this cyclic twinning and detwinning is essential for accurately predicting the plastic strain that occurs during fatigue loading. These experimental results have been used to improve and parameterize the twin models used in the PRISMSPlasticity crystal plasticity module and to predict the unique stress-strain loop shape associated with twinning and detwinning in $\mathrm{Mg}$ alloys, as shown in Fig. 12. To predict LCF life behavior, a novel dislocation dynamics simulation method has been developed. ${ }^{126}$ The method is energy-based and tracks the energy stored and dissipated in dislocation networks during cyclic deformation using ParaDiS.

Another important demonstration problem is the growth of small fatigue cracks as they advance across grain boundaries in $\mathrm{Mg}$ alloys. A unique ultrasonic fatigue scanning electron microscope (UFSEM) was used to quantify the growth rates of cracks as they advance through grains and across grain boundaries. ${ }^{127,128}$ Focused ion beam notches were placed in grain neighborhoods with specific characteristics (e.g., Schmid factors, misorientation). Detailed analysis of crack initiation lifetime, small crack propagation rates, crack interactions at grain boundaries and crack pathways in each grain were characterized, ${ }^{129}$ an example of which is shown in Fig. 12. High-energy x-ray tomography and diffraction have also been used to characterize the 3D crack path trajectory. ${ }^{129}$ These will be used in conjunction with a new fracture model being implemented in the PRISMS-Plasticity code to understand and simulate the influence of microstructure on small fatigue crack growth behavior.

These results were simulated using a new crystal elasticity and cyclic cohesive zone model for prediction of microstructural crack trajectories under high cycle fatigue ${ }^{130,131}$ or, in selected $2 \mathrm{D}$ cases, variational multi-scale modeling. ${ }^{73,132}$ The parameters of the cohesive law for crack propagation along various slip systems have been obtained using experimental (micro-beach marking) measurements of local crack growth rates within a grain. ${ }^{127}$ This is coupled with

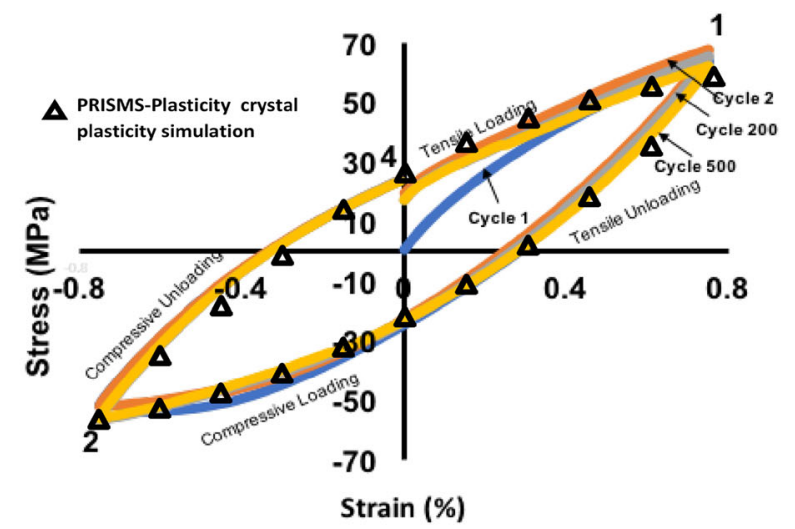

(a)

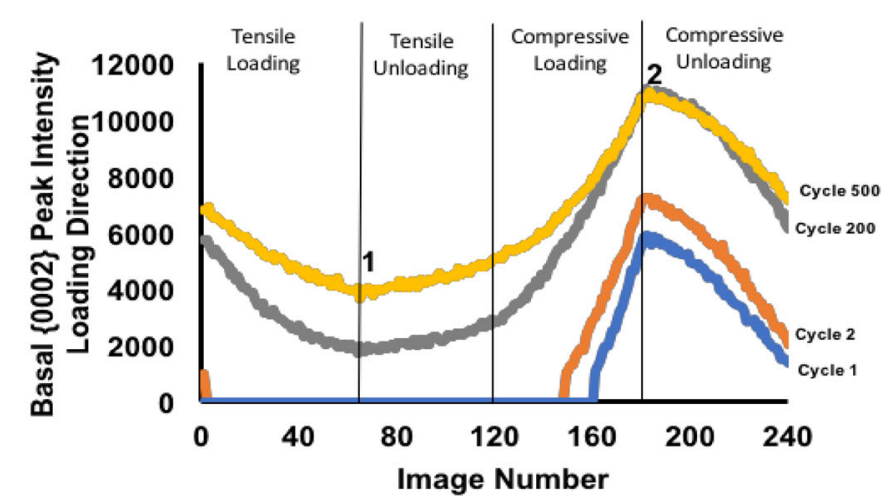

(b)

Fig. 12. (a). Cyclic stress-strain loops and corresponding $x$-ray peak intensity of the basal $\{0002\}$ peak for tests conducted at various points in the fatigue life of a pure Mg sample. PRISMS-Plasticity CPFE simulation results for cycle 2 ( $\Delta$ symbol) are also shown. (b) HEXD peak intensities in the loading direction indicate the onset of twinning and detwinning. 

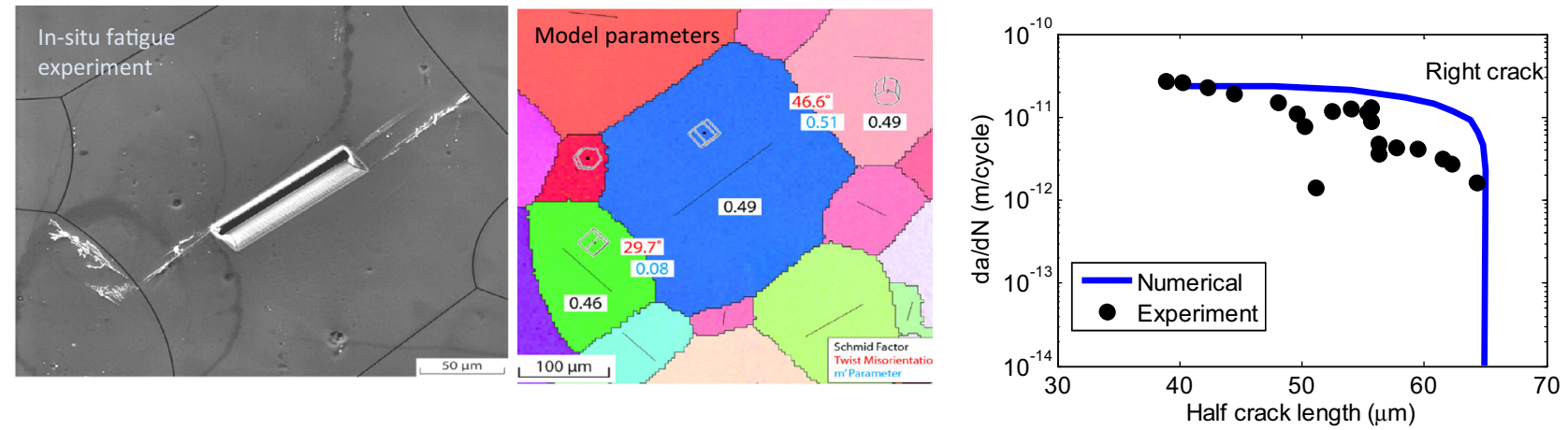

Fig. 13. Analytical model for predicting blocking versus transmission of cracks across a grain boundary. UFSEM experimental results (left) show that the left crack grows across the grain boundary while the right crack is blocked. Numerical results for the blocked crack are shown (right) with model parameters indicated (middle).

an analytical model for the effect of grain boundaries on crack paths, including prediction of grain boundary blocking or transmission of cracks under prescribed external loading based on tilt and twist angles made by the crack planes with respect to the grain boundary. ${ }^{131}$ The grain boundary effect is included in the fatigue crack growth model. ${ }^{130,131}$ An example of the simulation and experiments is shown in Fig. 13.

\section{Establishing the PRISMS Community}

We have engaged in substantial outreach to establish the external PRISMS community, an essential function of the PRISMS Center. An important component of our outreach is the PRISMS Workshop, which has been held annually since 2014. This workshop includes a technical component and a training component. We attract international leaders in the field of multi-scale modeling and magnesium research to attend and present at this workshop. To date, over 150 researchers have attended this workshop, and over 100 researchers have attended a two- or three-day PRISMS Software training workshop. Many members of this growing community are becoming external collaborators with the PRISMS Center, either using or contributing to open-source codes or involved in collaborations.

\section{Future Directions}

This article provides an overview of the PRISMS Center progress and activities. New integrated open-source, multi-scale PRISMS Software has been developed and made available to the broader community. A collaboration platform and information repository, the Materials Commons, has also been developed and made available to this community. All of these tools are continuously being improved and new versions released. These tools have been integrated into a framework of external software and experimental research to demonstrate accelerated development of fundamental understanding of thermodynamics and kinetics of precipitation and the effect of precipitate microstructure on tensile and fatigue properties of structural materials using $\mathrm{Mg}-\mathrm{RE}$ alloys as a model system. Future directions include significant upgrades to all software tools and the Materials Commons. Our Use Cases will shift toward developing predictive capabilities for the combined effects of grain size and solutes on tensile strength, alloying and microstructural effects on corrosion and fatigue behavior, and complex alloy optimization of structural $\mathrm{Mg}$ alloys.

The elements of the PRISMS Center comprise a comprehensive framework that can be used for accelerating a wide variety of materials developments. Although the PRISMS Center is focused on developing and demonstrating this capability for structural metals, elements of this capability can also be applied to energy and electronic materials. Many functional properties rely on or are strongly affected by the chemo-mechanical interactions that can be studied and predicted with the integrated multi-scale tools and framework of the Center. The PRISMS framework is being designed to enable rapid insertion of the most recent scientific advances into next-generation ICME capabilities and fulfill the vision of the Materials Genome Initiative.

\section{ACKNOWLEDGEMENTS}

This work was supported by the U.S. Department of Energy, Office of Basic Energy Sciences, Division of Materials Sciences and Engineering under Award\#DE-SC0008637 as part of the Center for Predictive Integrated Structural Materials Science (PRISMS Center) at University of Michigan. We also acknowledge the financial cost-share support of University of Michigan College of Engineering and Office of the Vice President for Research. The PRISMS Center supports methods development and software engineering in CASM. Methodology related to effective Hamiltonians to be implemented in future versions of CASM is supported by the National Science Foundation under Awards DMR1410242, DMR-1436154 and OAC-1642433. The 
DFT-FE software development has in major part been supported by DoE-BES with the pseudopotential part of the work supported by the PRISMS Center and the all-electron part of the work supported by DE-SC0017380.

\section{REFERENCES}

1. P. Giannozzi, S. Baroni, N. Bonini, M. Calandra, R. Car, C. Cavazzoni, D. Ceresoli, G.L. Chiarotti, M. Cococcioni, I. Dabo, A. Dal Corso, S. Fabris, G. Fratesi, S. de Gironcoli, R. Gebauer, U. Gerstmann, C. Gougoussis, A. Kokalj, M. Lazzeri, L. Martin-Samos, N. Marzari, F. Mauri, R. Mazzarello, S. Paolini, A. Pasquarello, L. Paulatto, C. Sbraccia, S. Scandolo, G. Sclauzero, A.P. Seitsonen, A. Smogunov, P. Umari, and R.M. Wentzcovitch, J. Phys. Condens. Matter 21,395502 (2009).

2. National Science and Technology Council, Materials Genome Initiative for Global Competitiveness (Executive Office of the President, Washington, DC, 2011). http://www.wh itehouse.gov/sites/default/files/microsites/ostp/materials_g enome initiative-final.pdf. Accessed 1 Mar 2016.

3. J. Allison, M. Li, C. Wolverton, and X. Su, JOM 58(11), 28 (2006).

4. D. Backman, D.Y. Wei, D.D. Whitis, M.B. Buczek, P.M. Finnigan, and D. Gao, JOM 58(11), 36 (2006).

5. T. Pollock, J.E. Allison, D.G. Backman, M.C. Boyce, M. Gersh, E. Holm, R. LeSar, M. Long, A.C. Powell, J.J. Schirra, D.D. Whitis, and C. Woodward, Integrated Computational Materials Engineering: A Transformational Discipline for Improved Competitiveness and National Security (Washington DC: National Academies Press, 2008).

6. W. Xiong and G. Olson, npj Comp. Mater. 2, 1 (2016).

7. S. Kalidindi, A. Medford, and D. McDowell, JOM 69, 2126 (2016).

8. M.F. Horstemeyer, Integrated Computational Materials Engineering (ICME) for Metals: Reinvigorating Engineering Design with Science (New York: Wiley, 2012).

9. H. Lim, F. Abdejljawad, S. Owen, B. Hanks, J. Foulk, and C. Battaile, Model. Simul. Mater. Sci. Eng. 24, 1 (2016).

10. The Minerals, Metals and Materials Society, Modeling Across Scales: A Roadmapping Study for Connecting Materials Models and Simulations Across Length and Time Scales (Pittsburgh: TMS, 2015).

11. The Minerals, Metals and Materials Society, Building a Materials Data Infrastructure: Opening New Pathways to Discovery and Innovation in Science and Engineering (Pittsburgh: TMS, 2015).

12. J. Segurado, R. Lebensohn, J. Llorca, and C. Tome, Int. J. Plast. 28, 124 (2012).

13. R. Lebensohn, A. Kanjarla, and P. Eisenlohr, Int. J. Plast. 32-22, 59 (2012).

14. H. Zhang, M. Diehl, F. Roters, and D. Raabe, Int. J. Plast. 80, 111 (2016).

15. A. van de Walle, R. Sun, Q. Hong, and S. Kadkhodaei, CALPHAD 58, 70 (2017).

16. M.R. Tonks, D. Gaston, P.C. Millett, D. Andrs, and P. Talbot, Comput. Mater. Sci. 51, 20 (2012).

17. J.E. Guyer, D. Wheeler, and J.A. Warren, Comput. Sci. Eng. 11, 6 (2009).

18. A. Jokisaari, P. Vorhees, J. Guyer, J. Warren, and O. Heinonen, Comput. Mater. Sci. 126, 139 (2017).

19. W. Bangerth, R. Hartmann, and G. Kanschat, ACM Trans. Math. Softw. 33, 24 (2007).

20. J. Bhattacharya and A. Van der Ven, Acta Mater. 56, 4226 (2008).

21. D. De Fontaine, Solid State Phys. 47, 33 (1994).

22. K.M. Rabe and U.V. Waghmare, Phys. Rev. B 52, 13236 (1995).

23. J.M. Sanchez, F. Ducastelle, and D. Gratias, Phys. A 128, 334 (1984).

24. J.C. Thomas and A. Van der Ven, Phys. Rev. B 88, 214111 (2013).
25. A. Van der Ven, J.C. Thomas, Q. Xu, and J. Bhattacharya, Math. Comp. Sim. 80, 1393 (2010).

26. U.V. Waghmare and K.M. Rabe, Phys. Rev. B 55, 6161 (1997).

27. W. Zhong, D. Vanderbilt, and K.M. Rabe, Phys. Rev. Lett. 73, 1861 (1994).

28. W. Zhong, D. Vanderbilt, and K.M. Rabe, Phys. Rev. B 52, 6301 (1995).

29. B. Puchala and A. Van der Ven, Phys. Rev. B 88, 094108 (2013).

30. G. Kresse and J. Hafner, Phys. Rev. B 47, 558 (1993).

31. G. Kresse and J. Hafner, Phys. Rev. B 49, 14251 (1994).

32. G. Kresse and J. Furthmüller, Phys. Rev. B 54, 11169 (1996).

33. G. Kresse and J. Furthmüller, Comput. Mat. Sci. 6, 15 (1996).

34. P. Giannozzi, O. Andreussi, T. Brumme, O. Bunau, M. Buongiorno Nardelli, M. Calandra, R. Car, C. Cavazzoni, D. Ceresoli, M. Cococcioni, N. Colonna, I. Carnimeo, A. Dal Corso, S. de Gironcoli, P. Delugas, R.A. DiStasio Jr, A. Ferretti, A. Floris, G. Fratesi, G. Fugallo, R. Gebauer, U. Gerstmann, F. Giustino, T. Gorni, J. Jia, M. Kawamura, H.-Y. Ko, A. Kokalj, E. Küçükbenli, M. Lazzeri, M. Marsili, N. Marzari, F. Mauri, N.L. Nguyen, H.-V. Nguyen, A. Otero-de-la-Roza, L. Paulatto, S. Poncé, D. Rocca, R. Sabatini, B. Santra, M. Schlipf, A.P. Seitsonen, A. Smogunov, I. Timrov, T. Thonhauser, P. Umari, N. Vast, X. Wu, and S. Baroni, J. Phys. Condens. Matter. 29, 465901 (2017).

35. G.L.W. Hart, V. Blum, M.J. Walorski, and A. Zunger, Nat. Mater. 4, 391 (2005).

36. L.J. Nelson, G.L.W. Hart, F. Zhou, and V. Ozolinš, Phys. Rev. B 87, 035125 (2013).

37. F. Pedregosa, G. Varoquaux, A. Gramfort, V. Michel, B. Thirion, O. Grisel, M. Blondel, P. Prettenhofer, R. Weiss, V. Dubourg, J. Vanderplas, A. Passos, D. Cournapeau, M. Brucher, M. Perrot, and E. Duchesnay, J. Mach. Learn. Res. 12, 2825 (2011).

38. F.-A. Fortin, F.-M. De Rainville, M.-A. Gardner, M. Parizeau, and C. Gagné, J. Mach. Learn. Res. 13, 2171 (2012).

39. J.C. Thomas and A. Van der Ven, Phys. Rev. B 90, 224105 (2014).

40. A.R. Natarajan, J.C. Thomas, B. Puchala, and A. Van der Ven, Phys. Rev. B 96, 134204 (2017).

41. A. Van der Ven, G. Ceder, M. Asta, and P.D. Tepesch, Phys. Rev. B 64, 184307 (2001).

42. A. Van der Ven and G. Ceder, Phys. Rev. Lett. 94, 045901 (2005).

43. A. Van der Ven, J.C. Thomas, Q. Xu, B. Swoboda, and D. Morgan, Phys. Rev. B 78, 104306 (2008).

44. Q. Xu and A. Van der Ven, Intermetallics 17, 319 (2009).

45. J. Bhattacharya and A. Van der Ven, Phys. Rev. B 81, 104304 (2010).

46. A. Van der Ven, H.-C. Yu, G. Ceder, and K. Thornton, Progress. Mater. Sci. 55, 61 (2010).

47. Q. Xu and A. Van der Ven, Phys. Rev. B 81, 064303 (2010).

48. J. Bhattacharya and A. Van der Ven, Phys. Rev. B 83, 144302 (2011).

49. A. Van der Ven, J. Bhattacharya, and A.A. Belak, Accounts Chem. Res. 46, 1216 (2010).

50. B. Puchala, M.L. Falk, and K. Garikipati, J. Chem. Phys. 132,134104 (2010).

51. S. DeWitt, S. Rudraraju, K. Thornton (2018, in preparation).

52. S. DeWitt, E.L.S. Solomon, A.R. Natarajan, V. AraulloPeters, S. Rudraraju, L.K. Aagesen, B. Puchala, E.A. Marquis, A. Van der Ven, K. Thornton, and J. Allison, Acta Mater. 36, 378 (2017).

53. S. DeWitt, A.R. Natarajan, A. Van der Ven, K. Thornton K, J. Allison (2018, in preparation).

54. S. DeWitt, C. Yang, L. Qi, J. Allison (2018, in preparation).

55. A. Jokisaari, C. Permann, and K. Thornton, Comput. Mater. Sci. 112, 128 (2016).

56. D. Montiel, J. Luce, S. Rudraraju, S. DeWitt, K. Thornton (2018, in preparation). 
57. C. Burstedde, L.C. Wilcox, and O. Ghattas, SIAM J. Sci. Comp. 33, 1103 (2011).

58. M. Kronbichler and K. Kormann, Comp. Fluids 63, 135 (2012).

59. https://nanohub.org/infrastructure/rappture.

60. V. Sundararaghavan and N. Zabaras, Inter. J. Plast. 24, 1581 (2008).

61. A. Kumar and V. Sundararaghavan, Finite Elements Anal. Design 127, 1 (2017).

62. V. Sundararaghavan, A. Kumar, and S. Sun, Acta Mater. 93, 12 (2015).

63. S. Sun and V. Sundararaghavan, Int. J. Solids Struct. 51, 3350 (2014).

64. C.N. Tomé, R.A. Lebensohn, and U.F. Kocks, Acta Metal. Mater. 39, 2667 (1991).

65. S. Ganesan, Microstructural Response of Magnesium Alloys: 3D Crystal Plasticity and Experimental Validation, Ph.D. Thesis, University of Michigan Ann Arbor, 2017.

66. S. Sun, A. Ramazani, and V. Sundararaghavan, Metals 7, 345 (2017).

67. A. Roy, S. Ganesan, S. Gentry, A. Trump, K. Thornton, V. Sundararaghavan, J. Allison, unpublished research 2018.

68. P. Acar and V. Sundararaghavan, AIAA J. 54, 4022 (2016)

69. P. Acar, A. Ramazani, and V. Sundararaghavan, Metals 7, 459 (2017).

70. R. Liu, A. Kumar, Z. Chen, A. Agrawal, V. Sundararaghavan, and A. Choudhary, Sci. Rep. 5, 11551 (2015).

71. P. Motamarri, M.R. Nowak, K. Leiter, J. Knap, and V. Gavini, J. Comp. Phys. 253, 308 (2013).

72. P. Motamarri and V. Gavini, Phys. Rev. B 90, 115127 (2014).

73. P. Motamarri and V. Gavini, Phys. Rev. B 97, 165132 (2018).

74. S. Panwar, S. Sun, and V. Sundararaghavan, Eng. Fract. Mech. 162, 290 (2016).

75. K. Sagiyama, S. Rudraraju, and K. Garikipati, Comput. Meth. Appl. Mech. Engin. 311, 556 (2016).

76. K. Sagiyama, S. Rudraraju, and K. Garikipati, Unconditionally stable, second-order accurate schemes for solid state phase transformations driven by mechano-chemical spinodal decomposition. Comput. Methods Appl. Mech. Eng. 311, 556 (2016).

77. G. Teichert, S. Rudraraju, and K. Garikipati, A variational treatment of material configurations with application to interface motion and microstructural evolution. J. Mech. Phys. Solids 99, 338 (2017). https://doi.org/10.1016/j.jmps. 2016.11.008.

78. G. Teichert, H. Gunda, S. Rudraraju, A. Natarajan, B. Puchala, K. Garikipati, and A. Van der Ven, A comparison of Redlich-Kister polynomial and cubic spline representations of the chemical potential in phase field computations. Comput. Mater. Sci. 128, 127 (2017). https://doi.org/10.10 16/j.commatsci.2016.11.024.

79. S. Rudraraju, A. Van der Ven, and K. Garikipati, $n p j$ Comput. Mater. (2016). https://doi.org/10.1038/npjcompu mats.2016.12.

80. Z. Wang, S. Rudraraju, and K. Garikipati, J. Mech. Phys. Solids 94, 336 (2016). https://doi.org/10.1016/j.jmps.2016. 03.028 .

81. S. Rudraraju, A. Van der Ven, and K. Garikipati, Comput. Methods Appl. Mech. Eng. 278, 705 (2014).

82. K.R. Elder and M. Grant, Phys. Rev. E 70, 051605 (2004).

83. J. Luce and K. Thornton, "Parameterization of the Structural Phase Field Crystal (XPFC) model for the simulation of hexagonal close packed (HCP) grain boundary kinetics (in preparation).

84. M. Greenwood, J. Rottler, and N. Provatas, Phys. Rev. E 83, 031601 (2011)

85. M. Greenwood, N. Provatas, and J. Rottler, Phys. Rev. Lett. 105,045702 (2010).

86. C. Ni, et al., Scr. Mater. 109, 94 (2015).

87. J. Luce, B. Hodges, P. Goins, E. Holm, and K. Thornton, "Comparison of 2D Hexagonal grain boundary structure and energies obtained from Structural Phase Field Crystal (XPFC) and Molecular Dynamic (MD) simulations (in preparation).

88. B. Puchala, G. Tarcea, E.A. Marquis, M. Hedstrom, H.V. Jagadish, and J.E. Allison, JOM 68, 2035 (2016).

89. The Materials Commons Python API, 'materials-commons', version 0.7.3. https://pypi.python.org/pypi/materials-com mons. Accessed 15 Mar 2018.

90. The Materials Commons. https://github.com/materials-co mmons/. Accessed 15 Mar 2018.

91. A. Dima, S. Bhaskarla, C. Becker, M. Brady, C. Campbell, P. Dessauw, R. Hanisch, U. Kattner, K. Kroenlein, M. Newrock, A. Peskin, R. Plante, S.-Y. Li, P.-F. Rigodiat, G.S. Amaral, Z. Trautt, X. Schmitt, J. Warren, and S. Youssef, JOM 68, 2053 (2016). https://doi.org/10.1007/s11837-0162000-4.

92. B. Blaiszik, K. Chard, J. Pruyne, R. Ananthankrishnan, S. Tuecke, and I. Foster, JOM 68, 2045 (2016). https://doi.org/ 10.1007/s11837-016-2001-3.

93. Materials Data Facility "Data publication and data discovery. https://materialsdatafacility.org. Accessed 1 Mar 2016.

94. K.Y. Zheng, J. Dong, X.Q. Zeng, and W.J. Ding, Mater. Sci. Eng. A 44, 489 (2008).

95. J. Wang, J. Meng, D. Zhang, and D. Tang, Mater. Sci. Eng. A 78, 456 (2007).

96. F. Penghuai, P. Liming, J. Haiyan, C. Jianwei, and Z. Chunquan, Mater. Sci. Eng. A 486, 183 (2008).

97. X. Gao, S.M. He, X.Q. Zeng, L.M. Peng, W.J. Ding, and J.F. Nie, Mater. Sci. Eng. A 431, 322 (2006).

98. T. Honma, T. Ohkubo, K. Hono, and S. Kamado, Mater. Sci. Eng. A 395, 301 (2005).

99. T. Honma, T. Ohkubo, S. Kamado, and K. Hono, Acta Mater. 55, 4137 (2007)

100. J.F. Nie and B.C. Muddle, Acta Mater. 48, 1691 (2000).

101. J.F. Nie, X. Gao, and S.-M. Zhu, Scr. Mater. 53, 1049 (2005).

102. S.R. Agnew and J.F. Nie, Scr. Mater. 63, 671 (2010).

103. A.R. Natarajan, E.L.S. Solomon, B. Puchala, E.A. Marquis, and A. Van der Ven, Acta Mater. 108, 367 (2016).

104. J.F. Nie, Metall. Mater. Trans. A 43, 3891 (2012).

105. A.R. Natarajan and A. Van der Ven, Acta Mater. 124, 620 (2016)

106. A.R. Natarajan and A. Van der Ven, Phys. Rev. B. 95, 214107 (2017).

107. E.L.S. Solomon and E.A. Marquis, The Structure of $\beta$ " and $\beta$ ' in an Aged Mg-Nd Alloy.Magnesium Technology 2016, ed. A. Singh, K. Solanki, M.V. Manuel, and N. NeelamegghamThe Minerals, Metals \& Materials Series, (Cham: Springer, 2016),

108. E.L.S. Solomon, V. Araullo-Peters, J.E. Allison, and E.A. Marquis, Scr. Mater. 128, 14 (2017).

109. E.L.S. Solomon, T. Chan, A. Chen, B. Utall-Veroff, and E.A. Marquis, Aging behavior of $\mathrm{Mg}$ alloys containing $\mathrm{Nd}$ and Y. Magnesium Technology 2017, ed. K. Solanki, D. Orlov, A. Singh, and N. Neelameggham (Pittsburgh, PA: TMS; New York: Springer, 2017).

110. E.L.S. Solomon, E.A. Marquis (2018, in preparation).

111. S. DeWitt, A.R. Natarajan, A. Van der Ven, K. Thornton, J. Allison (2018, in preparation).

112. S. DeWitt, C. Yang, Z. Huang, A. Misra, L. Qi, J. Allison (2018, in preparation)

113. M. Iyer, B. Radhakrishnan, and V. Gavini, J. Mech. Phys. Solids 76, 260 (2015)

114. S. Das and V. Gavini, J. Mech. Phys. Solids 104, 115 (2017)

115. A. Arsenlis, W. Cai, M. Tang, M. Rhee, T. Oppelstrup, G. Hommes, T.G. Pierce, and V.V. Bulatov, Modell. Simul. Mater. Sci. Eng. 15, 553 (2007).

116. L.K. Aagesen, J. Miao, J. E. Allison, S. Aubry, A. Arsenlis, Metall. Mater. Trans. A, 1 (2018)

117. Z. Huang, C. Yang, L. Qi, J. Allison, A. Misra (2018, in preparation).

118. E.L.S. Solomon and E.A. Marquis, Mater. Lett. 216, 67 (2018). 
119. Z. Huang, J. Allison, and A. Misra, Sci. Rep. 8, 3570 (2018). 120. Z. Chen and S.H. Daly, Exper. Mech. 57, 115 (2016).

121. A.D. Kammers and S. Daly, Exper. Mech. 53, 1743 (2013).

122. A.D. Kammers and S. Daly, Exper. Mech. 53, 1333 (2013).

123. A. Githens, S. Ganesan, Z. Chen, J.E. Allison, V. Sundararaghavan, S. Daly (2018, in preparation).

124. J.J. Bhattacharyya, F. Wang, P.D. Wu, W.R. Whittington, H. El Kadiri, and S.R. Agnew, Int. J. Plasticity 81, 123 (2016).

125. A. Murphy, D. Pagan, A. Beaudoin, M. Miller, J. Allison (2018, in preparation).

126. C. Heinrich and V. Sundararaghavan, Corros. Rev. 35, 325 (2017).
127. J.F. Adams, J.E. Allison, and J.W. Jones, Inter. J. Fatigue 93, 372 (2016).

128. J. Geathers, C.J. Torbet, J.W. Jones, and S. Daly, Inter. J. Fatigue 70, 154 (2015).

129. J. Adams, Investigating Microstructual Effects on Short Crack Grown and Fatigue Life Behavior of WE43 Mg, Ph.D. Dissertation, University of Michigan, Ann Arbor, MI, 2018.

130. S. Panwar and V. Sundararaghavan, Mater. Sci. Eng. A 708, 395 (2017).

131. S. Panwar, J. Adams, J.E. Allison, J.W. Jones, and V. Sundararaghavan, Int. J. Fatigue 113, 401 (2018).

132. V. Sundararaghavan and S. Sun, Math. Probl. Eng. 2016, 1 (2016). 